\title{
FACTORES ASOCIADOS A DEPRESIÓN EN RESIDENTES DE MEDICINA FAMILIAR
}

Carolina Nennethsy Garza-Rincones ${ }^{1,2}$, Enrique Villarreal-Ríos ${ }^{1,3 *}$, Emma Rosa Vargas-Daza ', Liliana Galicia-Rodríguez y Lidia Martínez-González'

'Unidad de Investigación Epidemiológica y en Servicios de Salud, IMSS; ${ }^{2}$ Residencia de Medicina Familiar, Unidad de Medicina Familiar 16, IMSS; ${ }^{3}$ Escuela de Medicina, División de Ciencias de la Salud, Universidad del Valle de México, Campus Querétaro. Querétaro, Qro.

RESUMEN: Objetivo: Determinar los factores asociados a depresión en residentes de medicina familiar. Material y Métodos: Estudio transversal comparativo en residentes de medicina familiar utilizando el Inventario de Depresión de Beck se conformaron dos grupos: el grupo 1 residentes con depresión y el grupo 2 residentes sin depresión. Se estudiaron aspectos sociodemográficos, familiares, físicos, y organizacionales. El análisis estadístico incluyó chi cuadrada, Razón de momios, Regresión logística múltiple y Cálculo de la probabilidad $\left(1 / 1+\mathrm{e}^{-\mathrm{y}}\right)$. Resultados: En el análisis bivariado los hábitos alimenticios y las crisis paranormativas resultaron significativos $(\mathrm{p}<0.05)$. El modelo que mejor explicó la depresión en los residentes de medicina familiar (chi cuadrada $=20.37 \mathrm{y} \mathrm{p}=0.001$ ) lo integraron las variables: sexo, sedentarismo, remuneración económica y crisis paranormativas. La probabilidad más alta de presentar depresión es del $87 \%$ al ser hombre, cursar con crisis paranormativas, sedentarismo y considerar una remuneración adecuada. Conclusión: El modelo para explicar la depresión en residentes de medicina familiar incluye variables que son susceptibles de modificar.

Palabras clave: Depresión. Medicina familiar. Crisis.

\section{FACTORS ASSOCIATED WITH DEPRESSION IN FAMILY} MEDICINE RESIDENTS

ABSTRACT: Objective: Determine the factors associated with depression in family medicine residents. Material and methods: This was a comparative cross-sectional study of family medicine residents using the Beck Depression Inventory. Two groups were formed: group 1, residents with depression and group 2, residents without depression. Sociodemographic, family, physical and organizational aspects were studied. The statistical analysis included chi square, odds ratio, multiple logistic regression and the calculation of probability $\left(1 / 1+\mathrm{e}^{-\mathrm{y}}\right)$. Results: In the bivariate analysis, eating habits and non-normative crises were significant $(\mathrm{p}<0.05)$. The model that best explained depression in family medicine residents (chi square $=20.37$ and $\mathrm{p}=0.001$ ) were the variables gender, sedentariness, remuneration and non-normative crises. The highest probability of presenting depression was $87 \%$ by being male, having non-normative crises, sedentariness and considering an adequate remuneration. Conclusion: The model that explains depression in family medicine residents includes variables that are susceptible to modification. (Rev Mex Med Fam. 2018;5:137-41)

Corresponding author: Enrique Villarreal-Ríos, enriquevillarrearios@gmail.com

Key words: Depression. Family medicine. Crisis.

\section{Correspondencia:}

*Enrique Villarreal-Ríos

Zaragoza y 5 de Febrero

Col. Centro

C.P. 26000, Querétaro, Qro., México

Fecha de recepción: 01-05-2018

E-mails: enriquevillarrearios@gmail.com

Fecha de aceptación: 27-06-2018

y enrique.villarreal@imss.gob.mx

Doi: $10.24875 / R M F .18000083$ 


\section{INTRODUCCIÓN}

La depresión como problema de salud mental es una de las principales causas de discapacidad. Se estima que afecta a cerca de 350 millones de personas en el mundo ${ }^{1}$.

El trastorno depresivo grave se caracteriza por una combinación de síntomas que interfieren con la capacidad para trabajar, dormir, estudiar, comer y disfrutar de las actividades que antes resultaban placenteras. Se atribuye mayor discapacidad a los trastornos mentales que a los físicos ${ }^{2,3}$.

Se ha calculado que la prevalencia de depresión es mayor entre los médicos residentes un $10 \%$ más que en la población general. La depresión es el desorden psiquiátrico más común en las mujeres médicas ${ }^{4}$ y se estima que los residentes que cursan con depresión tienen una tasa de error seis veces mayor en sus actividades y atención médica que los que no la presentan ${ }^{5}$.

La residencia médica es un periodo de formación con un elevado nivel de exigencia académica, actualización y autocrítica; caracterizado por enfrentamiento con situaciones emocionalmente impactantes; empobrecimiento de las relaciones familiares y de pareja; y con escaso tiempo para las actividades recreativas, escenarios todos ellos que pueden asociarse con depresión ${ }^{6,7}$.

La salud mental individual está determinada por múltiples factores sociales, psicológicos y biológicos ${ }^{8}$. Al tratarse de una entidad multifactorial, se ve afectada o favorecida de diferentes formas en las personas, por lo que el objetivo de este estudio es determinar los factores asociados para presentar depresión en los residentes de Medicina Familiar.

\section{MATERIAL Y MÉTODOS}

Se realizó un estudio transversal comparativo en residentes de Medicina Familiar de la Unidad de Medicina Familiar 16, de Querétaro (Qro.) durante el año 2016.

Se incluyeron a los residentes que estaban dispuestos a participar y que contestaron la encuesta en su totalidad.
Se integraron dos grupos: el grupo 1 estuvo compuesto por 16 residentes con depresión y el grupo 2 estuvo conformado por 26 residentes sin depresión. La depresión se determinó con la escala de Beck para la depresión ${ }^{9,10}$. Los grupos se integraron a partir del resultado del cuestionario de Beck, empleando como puntos de referencia: de 0 a 13 puntos, sin depresión; y de 14 a 63 puntos, presencia de depresión. Este instrumento tiene un a de Cronbach de 0.83 .

Se trabajó con el universo existente, por lo que no existió cálculo de tamaño de muestra ni técnica muestral.

Se estudiaron variables sociodemográficas (sexo y lugar de origen), aspectos familiares (estado civil, número de hijos, crisis paranormativas - presentes o ausentes en el momento de aplicar el formulario-), historia familiar conflictiva, determinada mediante un interrogatorio directo sobre aspectos físicos (tiempo de descanso en horas al día - horas de sueño reparador de 6-8 h-), hábitos alimentarios (definidos como buenos o malos de acuerdo al plato del buen comer) y sedentarismo (actividad física de por lo menos $150 \mathrm{~min}$ a la semana $)^{11}$, y aspectos organizacionales (año de residencia, instalaciones inadecuadas - opinión en torno a lo inadecuado o adecuado de las instalaciones- y remuneración recibida - opinión en torno a lo inadecuado o adecuado de la remuneración económica-).

El plan de análisis estadístico incluyó promedios, porcentajes, prueba de $\chi^{2}$, razón de momios, regresión logística múltiple y cálculo de la probabilidad $\left(1 / 1+\mathrm{e}^{-\mathrm{y}}\right)$.

En este trabajo no existen conflicto de intereses.

\section{RESULTADOS}

En el grupo con depresión la prevalencia del sexo femenino fue del $50.0 \%$ y en el grupo sin depresión la prevalencia del sexo femenino era del $69.2 \%$. En la tabla 1 se presentan otros datos sociodemográficos. 
Tabla 1. Factores asociados a depresión en residentes Medicina Familiar

\begin{tabular}{|c|c|c|c|c|c|c|c|}
\hline \multirow{2}{*}{ Factor asociado } & \multicolumn{2}{|c|}{ Porcentaje } & \multirow[b]{2}{*}{$\chi^{2}$} & \multirow[b]{2}{*}{$\mathbf{p}$} & \multirow[b]{2}{*}{ RM } & \multicolumn{2}{|c|}{ IC 95\% } \\
\hline & Depresión & No depresión & & & & Inferior & Superior \\
\hline \multicolumn{8}{|l|}{ Aspectos sociodemográficos } \\
\hline Sexo femenino & 50.0 & 69.2 & 1.55 & 0.21 & 0.44 & 0.12 & 1.60 \\
\hline Solteros & 68.8 & 61.5 & 0.22 & 0.63 & 1.37 & 0.36 & 5.14 \\
\hline Foráneos & 81.2 & 88.5 & 0.42 & 0.51 & 0.56 & 0.09 & 3.21 \\
\hline \multicolumn{8}{|l|}{ Aspectos familiares } \\
\hline Crisis paranormativas presentes & 68.8 & 26.9 & 7.07 & 0.008 & 5.97 & 1.52 & 23.42 \\
\hline Historia familiar conflictiva & 43.8 & 11.5 & 5.66 & 0.017 & 5.96 & 1.25 & 28.28 \\
\hline Con hijos & 25.0 & 23.1 & 0.20 & 0.88 & 1.11 & 0.26 & 4.75 \\
\hline \multicolumn{8}{|l|}{ Aspectos físicos } \\
\hline Malos hábitos alimenticios & 100.0 & 38.5 & 15.90 & 0.00 & - & - & - \\
\hline Sedentarismo & 75.0 & 42.3 & 1.11 & 0.29 & 1.92 & 0.56 & 6.51 \\
\hline Descanso de 5 h o menos & 12.5 & 23.1 & 0.71 & 0.39 & 0.47 & 0.08 & 2.71 \\
\hline \multicolumn{8}{|l|}{ Aspectos organizacionales } \\
\hline Instalaciones inadecuadas & 75.0 & 53.8 & 1.87 & 0.17 & 2.57 & 0.66 & 10.10 \\
\hline Remuneración inadecuada & 81.2 & 57.7 & 2.47 & 0.11 & 3.17 & 0.72 & 13.91 \\
\hline
\end{tabular}

IC 95\%: intervalo de confianza al 95\%; RM: razón de momios; ×2: chi cuadrada.

En el análisis bivariado, los hábitos alimenticios presentaron la mayor significancia estadística $\left(\chi^{2}=15.90 ; p=0.000\right)$, aunque las crisis paranormativas también resultaron estadísticamente significativas: en el grupo con depresión, el $68.8 \%$ mencionó cursar con este tipo de crisis y en el grupo sin depresión, el 26.9\% $\left(\chi^{2}=7.07 ; \mathrm{p}=0.008\right)$. En la tabla 1 se presentan los valores para el resto de los factores asociados estudiados.

En el análisis múltiple, el modelo que mejor explicóla presencia de depresión ( $\mathrm{p}$ ) enlos residentes de Medicina Familiar $\left(\chi^{2}=20.37\right.$; $\mathrm{p}=0.00)$ lo integraron las siguientes variables: sexo (masculino), sedentarismo (presente), remuneración económica (inadecuada) y crisis paranormativas (presentes). En la tabla 2 se presentan los valores de cada una de las variables.

La probabilidad más alta de presentar depresión es del $87 \%$ al ser hombre, cursar con crisis paranormativas, llevar un estilo de vida sedentario y considerar recibir una remuneración inadecuada. En la tabla 3 se presentan las probabilidades para cada uno de los escenarios estudiados.

\section{DISCUSIÓN}

La residencia de especialidad médica es un periodo de grandes cambios para los médicos que la realizan, que empieza con el estrés previo a la aplicación del examen nacional y continúa una vez que ingresan en el curso de especialización; en él, además, la demanda de tiempo y carga académica aumentan considerablemente, un escenario en el que el individuo se vuelve más susceptible, por lo que identificar los factores asociados a la depresión en los residentes de Medicina Familiar es un tema importante, de ahí la trascendencia del artículo que aquí se presenta.

Hay que reconocer que una limitante de este estudio es el tamaño de muestra. Al respecto, los investigadores están seguros de que este escenario aumenta la probabilidad de cometer el error tipo II; sin embargo, los resultados lo refutan en buena medida, aunque la posibilidad se encuentra latente 
Tabla 2. Modelo de regresión logística simple para explicar la depresión

\begin{tabular}{|l|c|c|c|}
\cline { 2 - 4 } \multicolumn{1}{c|}{} & \multicolumn{2}{c|}{ Chi cuadrada } & \multicolumn{2}{c|}{ p } \\
\cline { 2 - 4 } \multicolumn{1}{c|}{} & \multicolumn{2}{c|}{20.37} & Estadístico \\
\hline Factor asociado & Coeficiente & & p \\
\hline Constante & -2.927 & 4.38 & 0.036 \\
\hline Sexo (masculino) & -2.144 & 5.62 & 0.018 \\
\hline Sedentarismo (presente) & 2.542 & 2.28 & 0.130 \\
\hline Remuneración económica (inadecuada) & 1.452 & 6.98 & 0.008 \\
\hline Crisis paranormativas (presentes) & 2.367 & & \\
\hline
\end{tabular}

Tabla 3. Probabilidad de presentar depresión en residentes de Medicina Familiar en relación con los factores asociados

\begin{tabular}{|l|c|c|l|l|}
\hline Probabilidad & Crisis paranormativas & Sedentarismo & Sexo & Remuneración \\
\hline 0.879 & Sí & Sí & Hombre & Adecuada \\
\hline 0.784 & Sí & Sí & Mujer & Inadecuada \\
\hline 0.744 & No & Sí & Hombre & Inadecuada \\
\hline 0.709 & Sí & No & Hombre & Inadecuada \\
\hline 0.460 & Sí & Sí & Mujer & Adecuada \\
\hline 0.405 & No & Sí & Hombre & Adecuada \\
\hline 0.364 & Sí & No & Hombre & Adecuada \\
\hline 0.254 & No & Sí & Mujer & Inadecuada \\
\hline 0.222 & Sí & No & Mujer & Inadecuada \\
\hline 0.186 & No & No & Hombre & Inadecuada \\
\hline 0.074 & No & Sí & Mujer & Adecuada \\
\hline 0.063 & Sí & No & Mujer & Adecuada \\
\hline 0.051 & No & No & Hombre & Adecuada \\
\hline 0.026 & No & No & Mujer & Inadecuada \\
\hline
\end{tabular}

para aquellas variables que resultaron no significativas.

De igual forma, la validez de los resultados depende en buena medida de la veracidad de las respuestas dadas por los entrevistados; si éstos no contestaron con la verdad, los resultados se invalidan.

De acuerdo a la literatura médica, en el sexo femenino se incrementa la tendencia de presentar depresión, cuya explicación contempla los aspectos biológicos y el estereotipo. En torno a los aspectos biológicos, se atribuye el evento a las hormonas reproductivas; aunque se desconoce el mecanismo exacto en que las hormonas sexuales predisponen a la depresión, está comprobado que éstas tienen receptores en el cerebro. De igual forma, esta predisposición se ha explicado en función del desarrollo emocional y el estereotipo femenino de dependencia, pasividad, cuidar de otros antes de sí misma y baja competencia ${ }^{12}$. A pesar de ello, en este trabajo los resultados no apoyan esta tendencia, pues el análisis bivariado niega la asociación con depresión; sin embargo, en el análisis múltiple el sexo masculino es el que mayor predisposición presenta. Tal vez la explicación debería contemplar las características de la mujer médica y en él señalar que contradice el estereotipo clásico asignado a este género. Pero con los datos aquí disponibles no existe la suficiente información 
para realizar la afirmación, pues esto es motivo de otra investigación.

La socialización primaria se da en el núcleo familiar, es el primer contacto de convivencia y es considerado uno de los sistemas más importantes para la salud y el mejoramiento de la calidad de vida ${ }^{13}$, es por ello que las crisis paranormativas, al tratarse de eventos inesperados, causan un impacto en el estado funcional de la familia. Estas crisis requieren un proceso de adaptación para ser capaz de mantener la funcionalidad y continuidad familiar; si los miembros de la familia no son capaces de comunicarse de forma adecuada y continuar con la dinámica familiar, se pueden convertir en una familia disfuncional ${ }^{14}$. Los resultados del estudio coinciden con la literatura médica e identifican las crisis paranormativas como un factor asociado a la depresión. Se podría suponer, entonces, que en los residentes de Medicina Familiar el soporte familiar tiene un peso importante para evitar la depresión, situación que tal vez no sea exclusiva de esta población, por lo que entonces se podría afirmar que la familia juega un papel importante en la estabilidad emocional del individuo.

La depresión, de acuerdo a la severidad, genera grandes cambios en el comportamiento de quien la padece, y uno de ellos son los hábitos alimenticios. En este estudio, el 100\% del grupo con depresión mencionó tener malos hábitos alimenticios, escenario que desde el punto de vista estadístico cancela la posibilidad de análisis.

El modelo múltiple que mejor explicó la depresión incluyó variables que en el análisis bivariado no resultaron significativas. Esto es una realidad estadística, pues la variable adopta un comportamiento diferente cuando se analiza sola o en conjunto con otro grupo de variables, de ahí la trascendencia de pasar de modelos bivariados a modelos múltiples.
El modelo que mejor explica la depresión contempla el género, las crisis paranormativas, el sedentarismo y la remuneración. En él se aprecian variables susceptibles de ser modificadas, lo cual ofrece un área de oportunidad para los profesores de la residencia de Medicina Familiar preocupados por la salud emocional de los residentes. Esta información es reforzada por la estimación de probabilidad, la cual especifica puntualmente los factores asociados que pueden modificarse.

En conclusión, se puede decir que existe un modelo para explicar la depresión en los residentes de Medicina Familiar, escenario a partir del cual se pueden realizar acciones concretas si se pretende ligar los resultados de investigación con la aplicación práctica de ellos.

\section{BIBLIOGRAFÍA}

1. Berenzon S, Lara MA, Robles R, Medina-Mora ME. Depresión: es tado del conocimiento y la necesidad de políticas públicas y planes de acción en México. Salud Publica Mex. 2013;55(1):74-80.

2. Instituto Nacional de Salud Mental. Depresión; 2009 [citado en septiembre de 2016]. Disponible en: https://www.nimh.nih.gov/ health/publications/espanol/depresion/depresion_38791.pdf

3. Wagner F, González C, Sánchez S, García C, Gallo J. Enfocando la depresión como problema de salud pública en México. Salud Mental. 2012;35:3-11.

4. Mascarúa-Lara E, Vázquez-Cruz E, Córdova-Soriano JA. Ansiedad y depresión en médicos residentes de la especialidad en Medicina Familiar. Aten Fam. 2014;21(2).

5. Fahrenkopf AM, Sectish TC, Barger LK, Sharek PJ, Lewin D, Chiang VW, et al. Rates of medication errors among depressed and burnt out residents: prospective cohort study. BMJ. 2008;336(7642):488-91.

6. Manterola D. Factores socioeconómicos y psicoafectivos y su influencia en el rendimiento académico de los residentes de Ginecología y Obstetricia. Ginecol Obstet Mex. 2015;83:139-47.

7. Mariños A, Otero M, Málaga G, Tomateo J. Coexistencia de síndrome de Burnout y síntomas depresivos en médicos residentes. Estudio descriptivo transversal en un hospital nacional de Lima. Rev Med Hered. 2011;22:162-8.

8. Montoya LM, Gutiérrez JA, Toro BE, Briñón MA, Rosas E, Salazar LE. Depresión en estudiantes universitarios y su asociación con el estrés académico. Rev CES Med 2010;24(1):7-17.

9. Beck A, Steer R, Brown G. Inventario de Depresión Beck. [acceso el 5 de noviembre de 2016]. Disponible en: http://gsdl.bvs. sld.cu/cgi-bin/library?e=d-00000-00---off-0psicolo1--00-0----010-0---0---0direct-10---4-------0-11--11-es-50---20-about---00-01-00-0-0-11-1-0utfZz-8-00\&a=d\&c=psicolo1\&cl=CL1\&d=HASH 01c8a63a59a94100eaf4f010.6.1.7

10. Sanz J, Vázquez C. Adaptación española del Inventario de Depresión Beck. Consejo General de Colegios Oficiales de Psicólogos. [acceso el 5 de noviembre de 2016]. Disponible en: https://www.cop.es/uploads/PDF/2013/BDI-II.pdf

11. Organización Mundial de la Salud. [citado en octubre de 2016 acceso el 14 de enero de 2017]. Disponible en: http://www.who. int/mediacentre/factsheets/fs385/es/

12. Zarragoitía I. Las diferencias de sexo y género en la depresión proclividad en la mujer. Medweve. 2013;13(3).

13. Vargas H. Tipo de familia y ansiedad y depresión. Rev Med Hered. 2014;25:57-9.

14. González I. Las crisis familiares. Rev Cubana Med Gen Integr 2000;16(3):280-6. 